\title{
Statistical Description and Analysis of the Concurrent Data Transmission from Massive MTC Devices
}

\author{
Xin Jian, Xiaoping Zeng, Jie Huang, Yunjian Jia and Yu Zhou \\ College of Communication Engineering, Chongqing University, China \\ jianxin_zg@163.com,jianxin@cqu.edu.cn
}

\begin{abstract}
The concurrent data transmission from massive machine type communications $(M T C)$ devices within a short time period makes the traffic flow of MTC more bursty. The elassic Markovian traffic models under the assumption of Poisson arrival, whose inter-arvival time (IAT) is a negative exponentially distributed random variable with infinite support range, are no longer suitable for this situation. Beta distribution, a distribution with finite support range, is then proposed by 3GPP to account for this and used to serve as the IAT of MTC. The researches on the traffic modeling based on Beta dis ribution is still in its very initial step and is rarely reported in state of the art literatures. This article takes the initiatives to discuss some prime issues for MTC traffic modeling under the assumption of Beta arrival. By the usage of renewal theory and Volterra integral equation of the second kind with difference kernel, we present the methodology to deduce the access intensity of MTC arrival process, which is defined as the mean number of nenewals by time to Numerical results show that in the context of MTC, there is a sharp increase in the number of access requests from MTC devices which directly causes the well-knowncongestion problem of MTC. And the ratio of $\beta$ and $\alpha$ of Beta $(\alpha, \beta)$ distribution to some extent can beused as a metric to represent the burstiness of Beta distribution or that of MTC. These works together provide researchers and engineers a basis to appropriately choose traffic models for different MTC applications.
\end{abstract}

Keywords: Machine type communications; Beta distribution; Access Intensity; Volterra integral equation of second kind with difference kernel

1. Introduction

Besides the call for higher data rates and more capacity to cope with the traffic prediction in the next few years, operators and manufactures in the $3^{\text {rd }}$ Generation Partnership Project (3GPP) also unanimously agreed on the continuous enhancement for machine type communications (MTC) and support for diverse traffic types [1]. MTC is defined as machine to machine communications over cellular mobile networks. It has a tremendous market potential and is an important revenue stream for operators $[2,3]$. Figare 1 depicts the typical network architecture and applications of MTC. The network consists of three components [3, 4], namely the MTC server(s), the communication network(s) and the huge amount of autonomous industrial MTC devices from smart metering like smart grid, smart home, Internet of vehicles (IoV), Internet of things (IoT), wireless sensor network (WSN) and etc. The possibility of these massive MTC devices generating their access attempts within a short time period makes the traffic characteristics of MTC quite different from the conventional network services [5]. Therefore, conventional cellular mobile networks that have been optimally designed for 
Human to Human $(\mathrm{H} 2 \mathrm{H})$ communications should be developed to accommodate these new challenges and guarantee the quality of service (QoS) of both MTC and H2H communications.

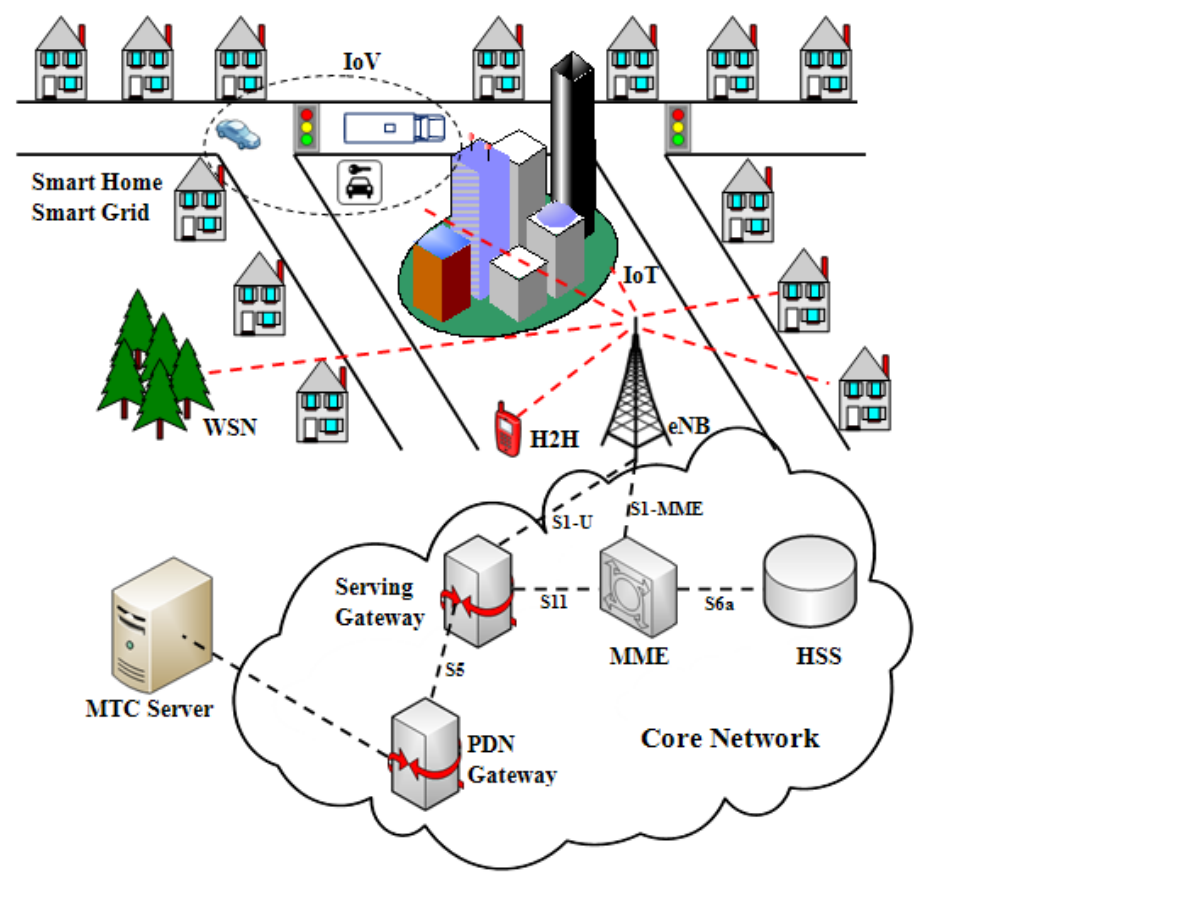

Figure 1. Typical Network Architecture and Application Scenarios of MTC

Prior to any technology enhancements for MTC, an appropriate traffic model enabling preliminary performance evaluation is of prime important. Classic Markovian traffic models under the assumption of Poisson arrival have been long-term used for stochastic traffic modeling in telecommunications [6]. These models include the simplest homogeneous Poisson proeess and the more complex compound Poisson process, such as the Markonarrival process and the Markov modulated Poisson process [6]. Compound Poisson process is a nature extension of homogeneous Poisson process. It assumes the arrival rate of Poisson process to be a random variable or random process rather than a constant. In recent publications, these models have been directly applied to MTC traffic modeling [7-10]. However, the concurrent data transmission from massive MTC devices within a short time period makes the arrival pattern more bursty and invalidates the Poisson assumption of these models. To capture this burstiness and evaluate the network performance under different access intensities, an appropriate statistical description of this burstiness should be concerned. As a major involver, 3GPP proposes two reference traffic models, in which the inter-arrival time (IAT) of MTC is assumed to follow uniform distribution or Beta distribution, respectively [11]. These two distributions correspond to two realistic scenarios when MTC devices access to the network in non-synchronized or synchronized manner. Uniform distribution is a special case of Beta distribution. Beta distribution is a distribution with two shape parameters and limited support range which has the flexibility to model a lot of MTC devices starting their access within a short time period. However, unlike the full understood Markovian traffic models, the researches on the traffic modeling based on Beta distribution is still in its very initial step and is rarely reported in state of the art literatures. 
In this paper, we take the initiatives to discuss some prime issues for MTC traffic modeling under the assumption of Beta arrival. In Chapter 2, we briefly introduce the typical traffic characteristics of MTC and the reason why Beta distribution is more suitable for MTC. Then by the usage of renewal theory and Volterra integral equation of the second kind with difference kernel, in Chapter 3, we provide the methodology to deduce the access intensity of MTC arrival process which is defined as the number of renewals by time $t$. Numerical results in Chapter 4 show that in the context of MTC, there is a sharp increase in the number of access requests from MTC devices which directly causes the well-known congestion problem of MTC that has been reported in [3, $5,11]$. These works provide researchers and engineers a basis to appropriately choose traffic models for different MTC applications.

\section{Typical Traffic Characteristics of MTC and Beta Distribution}

In the context of MTC, the service data to be communicated have been preprogrammed inside the MTC device and MTC server before the service is deployed. So compared with $\mathrm{H} 2 \mathrm{H}$ communications, MTC services haye less andomness. The sessions are always triggered by predefined time or events [10], as shown in Figure 2, in which $T$ is the predefined communication pertod that depends on specific applications and $T_{1}, T_{2}, \ldots$ are the inter-arrival time of random events triggering the MTC access requests or data transmission.
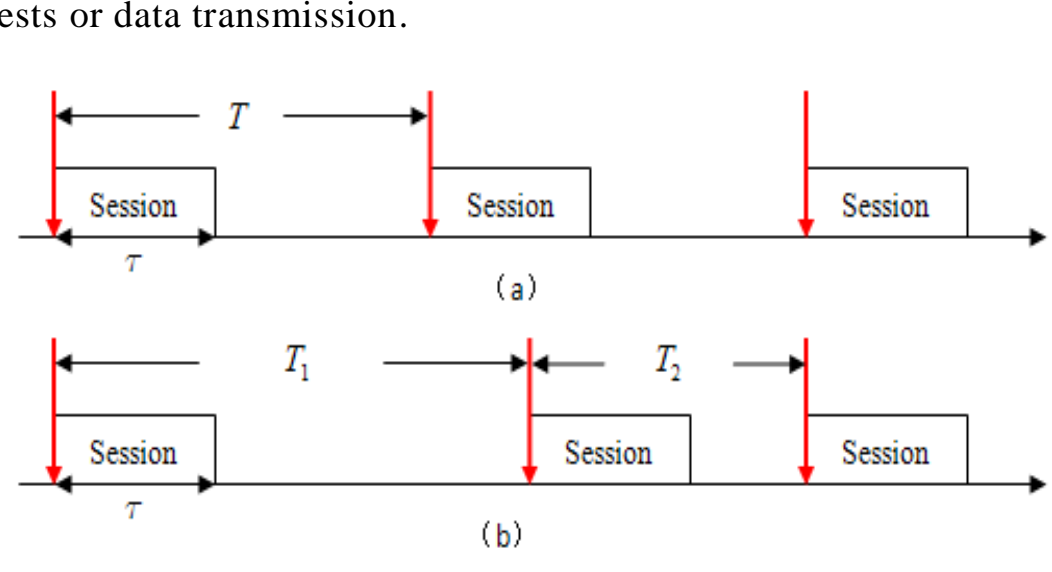

Figure 2. (a) Periodically Initiated Sessions; (b) Randomly Initiated Sessions

Usually, the average session length $t$ is quite smaller than $T$ or $T_{1}, T_{2}, \ldots$, that is, $t=T$ or $T_{1}, T_{2}, \ldots$. When massive MTC devices generate their access attempts within such a short time period, it would make the arrival pattern more bursty. As the interarrival time of Poisson process is a negatively exponential distributed random variable which bas infinite support range, it is no longer suitable for this situation. Thus 3GPP suggests a distribution with finite support range, namely Beta distribution, to capture this traffic characteristic of MTC. The probability density function (PDF) of Beta distribution, for $0 \leq t \leq \tau$ and shape parameters $\alpha>0$ and $\beta>0$, is a power function of the variable $t$ and of its reflection $(1-t)$, that is [11]

$$
f(t)=\frac{\Gamma(\alpha+\beta)}{\Gamma(\alpha) \Gamma(\beta)} \frac{t^{\alpha-1}(\tau-t)^{\beta-1}}{\tau^{\alpha+\beta-1}}
$$


where $\Gamma(\square)$ is the Gama function. As any spreading time $\tau$ can be normalized to unit one with the scale operation $y=x / \tau$, thus in the rest of this paper, the spreading time $\tau$ of Beta distribution is assumed to be unit one. When $\tau=1$, the mean and variance are [12]

$$
E[t]=\frac{\alpha}{\alpha+\beta}, \quad \operatorname{var}[t]=\frac{\alpha \beta}{(\alpha+\beta)^{2}(\alpha+\beta+1)}
$$

The mean of Beta distribution is a monotonous rising function of $\alpha$ and a monotonous decreasing function of $\beta$. The variance of Beta distribution is a monotonous decreasing function of $\alpha$ and $\beta$.

The PDFs of the negative exponential distribution and the Beta distribution are illustrated in Figure 3. The main differences are:

1) Beta distribution is a distribution with limited support range which is able to model a lot of MTC devices starting their access within a short time period, while exponential distribution extends itself to infinite range which is incapable of this.

2) Beta distribution is a concave function with a maximum within the support range (for shape parameters larger than one). This implies most of MTC devices would gather around at the mode (maximum value) of the PDF to transmit and behaves in a synchronized manner. While exponential distribution has no meaningful mode, it tends to distribute the access devices more evenly in the infinite time range. Therefore, to some extent, Beta distribution captures the burstiness of MTC and can be used to model the inter-arrivalime of MTC within one session.

So far, the missing link is the Qalidation of this work by measurements. When the field test results are available, the method proposed by Karl Pearson can be used to estimate the shape parameters of Betandistribution with sample mean and sample variance or higher moments 13$]$.

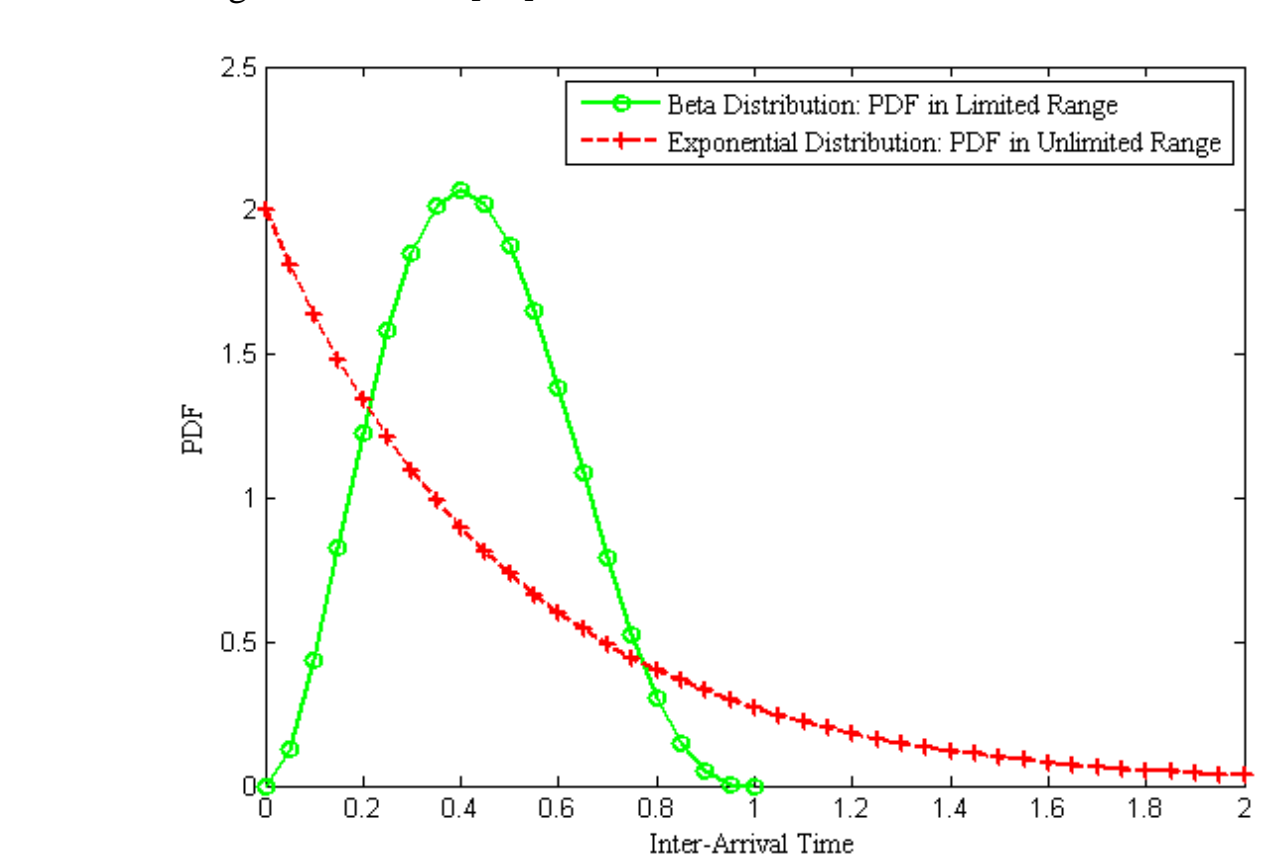

Figure 3. PDFs of Exponential Distribution and Beta Distribution 


\section{Access Intensity of the Arrival Process}

When the IAT of MTC follows Beta distribution, it is necessary to present the main features of the arrival process under this assumption. In this chapter, by the usage of renewal theory and Volterra integral equation of the second kind with difference kernel, we evaluate the access intensity of the arrival process, which is defined as the mean number of renewals by time $t$.

\subsection{Theoretical Analysis}

If the sequence of nonnegative random variables $X_{1}, X_{2}, \ldots$ is independent and identically distributed with a PDF $f(t)$, then the counting process $N(t), t \geq 0$ is said to be a renewal process. Poisson process is a special case of renewal process where $f(t)$ is exponentially distributed. In the context of MTC, $f(t)$ equals Beta distribation. In renewal theory, $N(t)$ represents the number of renewals by time $t$. When used in telecommunications, it represents the number of access requests of customers by time $t$, which can be defined as the access intensity of the arrial process

Theoretically, the distribution of $N(t)$ can be obtained. But as it involves n-fold convolution of $f(t)$ with itself, it is always impossible to calculate analytically expect for some special cases. Therefore, we compromise to evaluate the mean value of $N(t)$. Let $m(t)=E[N(t)]$, it can be shown that there is a one to one correspondence between the IAT distribution $f(t)$ and $m(t)$, that is [14]

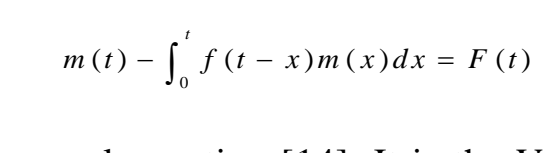

This is the well-known renewal equation [14]. It is the Volterra integral equation of the second kind with difference kernel [15]. The general solution process for Volterra integral equation of the second kind with difference kernel can be realized in the transform domain-15. Firstly applying the Laplace transform to Equation (3) and taking into account the fact that by the convolution theorem the integral with kernel depending on the difference the arguments is transformed into the product $f(s) m(s)$, we arrive at the following equation

in which $m(s), F(s)$ and $f(s)$ are the Laplace transform of $m(t), F(t)$ and $f(t)$, respectivery Then, $m(s)$ is given by

$$
m(s)=\frac{F(s)}{1-f(s)}
$$

As the right-hand function $F(t)$ is the integral of the kernel $f(t)$, that is,

$$
F(t)=\int_{0}^{t} f(x) d x
$$

According to the property of Laplace transform, $F(s)=f(s) / s$. The solution of Equation (5) can further be simplified as 


$$
m(s)=\frac{f(s)}{s(1-f(s))}
$$

Applying the Laplace inversion transform $m(t)$ can be obtained.

\subsection{Moment Generation Function of Beta Distribution}

To solve Equation (7), the first and most important task is to give out the Laplace transform of Beta distribution. As we know, the moment generation function (MGF) of PDF is defined as [14]

$$
M(u) @ \int_{-\infty}^{\infty} e^{u x} f(x) d x
$$

Thus, the Laplace transform of PDF $f(s)=M(-s)$. So we only have to calculate the MGF of Beta distribution.

The MGF of Beta distribution with shape parameters $\alpha$ and $\beta$ is the confluent hyper-geometric function of first kind with parameters $\alpha$ and $\alpha+\beta$, that is, ${ }_{1} F_{1}(\alpha, \alpha+\beta, u)$. [16] gives out its analytical expression. The work of deduction involves the following two propositions.

Proposition 1 Supposing $n$ is a positive integer, the confluent hyper-geometric function of first kind with parameters $a=1$ and $b=1+n$ canbe represented as [16]

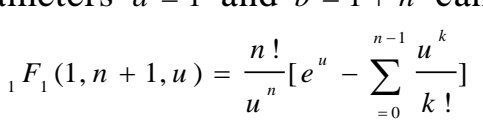

Proposition 2 (Derivative of $F_{1}(\ell, \mu)$ ) [17]

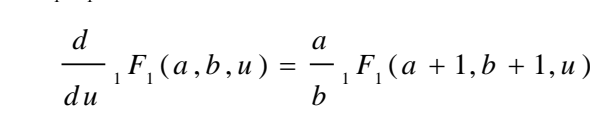

With Equation (9) and (10), the MGF of Beta distribution with any integral parameters can be solved as follows 16]:

1) Let $n=\beta$ and substitute itinto Equation (9), we obtain ${ }_{1} F_{1}(1, \beta+1, u)$;

2) Find the $(\alpha-1)$ th order derivative of ${ }_{1} F_{1}(1, \beta+1, u)$ and scale it with a constant resulting from Equation (10), we obtain ${ }_{1} F_{1}(\alpha, \alpha+\beta, u)$ that is the MGF of Beta distribution With shape parameters $\alpha$ and $\beta$.

In Table 1, we give out the MGFs of Beta distributions with low orders which will be analyzed in the rest of this paper. Besides 3GPP proposals, to choose shape parameters of Beta distribution for specific MTC applications, Karl Pearson's estimation method based on field tests will be helpful. Substituting these MGFs of Beta distributions into Equation (7), the access intensity of MTC in S-domain $m(s)$ can be obtained, then the $m(t)$.

\section{Numerical Analysis}

Though the analytical process to solve $m(t)$ has been given, the inverse Laplace transform of Equation (7) is always impossible. We have to resort to numerical Laurent series expansion for approximation. To do this, we notice that 


$$
f(0)=M(0)=1
$$

which implies $s=0$ is a second order pole of Equation (7). This provides us the feasibility to solve Equation (7) by means of numerical Laurent series expansion. Though this is not an analytical solution, it still gives us a general view on the trend of the access intensity of MTC under different inter-arrival time distributions.

Table 1. MGF of Low Order Beta Distributions

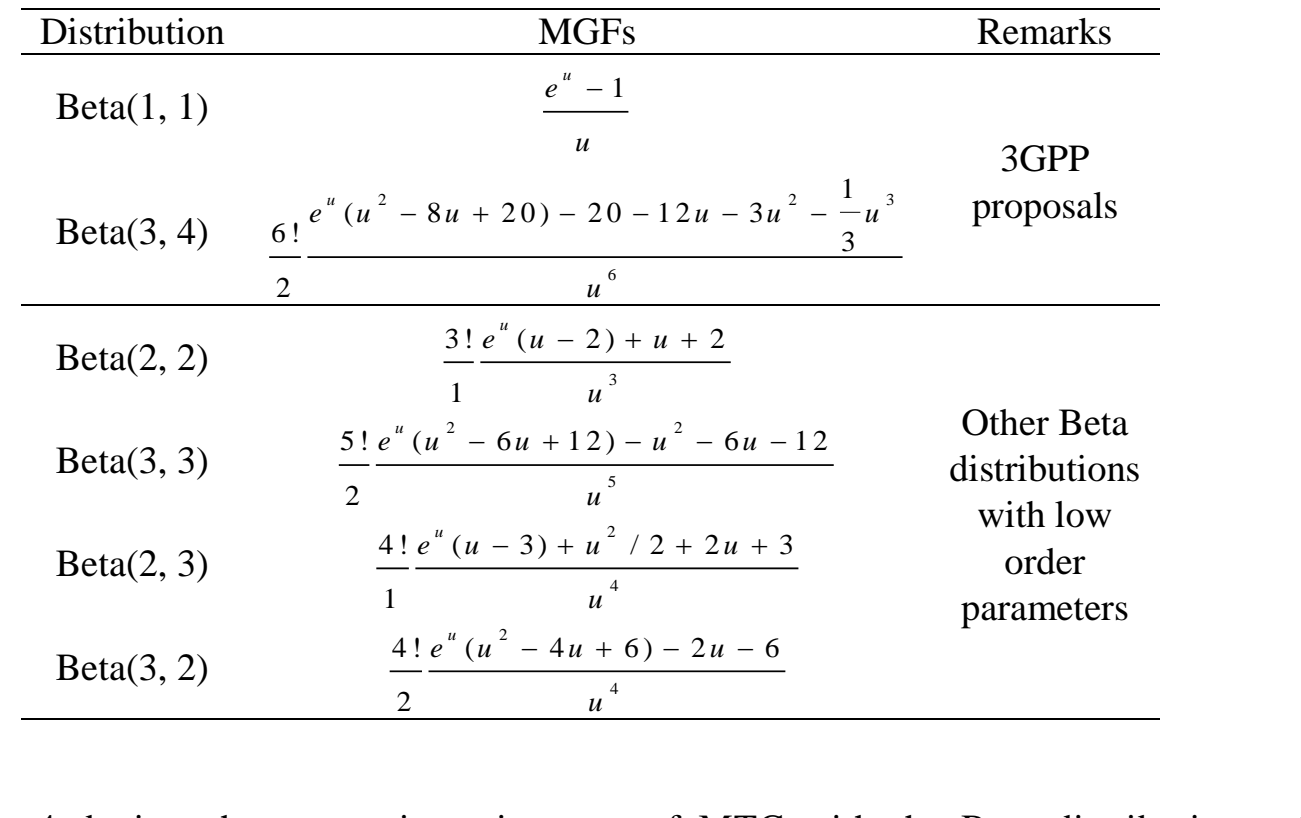

Figure 4 depicts the access intensit $m(t)$ of MTC with the Beta distributions of $3 \mathrm{GPP}$ proposals, that is $\mathrm{Beta}(1,1)$ and Beta $(3,4)$. It shows that:

1) If the arrival pattern is a Poisson arrival process, $m(t)$ is strictly linear with $\lambda$ as $m(t)=\lambda+$. n Figure 4 and Figure 5, we normalized $l$ to unit one.

2) If the arriyal pattern Collows uniform distribution, $m(t)$ increases faster than that of Poisson arriyal. This implies the mean number of customers requesting service within a time interval of uniform distribution will be greater than that of Poisson arrival.

$3)$ If the arrival pattern follows $\operatorname{Beta}(3,4), m(t)$ increases faster than that of Poisson arrival and uniform distribution. But at some time point, when the time interval is less than that point, $m(t)$ is less than that of Poisson arrival and uniform distribution. To some extent, this is because the cumulative density function of Beta distribution is less than that of exponential distribution within this time range.

Figure 5 depicts the access intensity $m(t)$ of MTC with lower order Beta distributions in Table 1. Without loss of generality, it shows the general trend of $m(t)$ under different Beta distributions with acceptable calculation complexity. It shows that: 
1) The curves of $m(t)$ of all Beta distributions, to some extent, follow the exponential increase trend and increase faster than that of Poisson arrival. But for the same reason mentioned above, at some point, they are less than that of Poisson arrival.

2) The curves of $m(t)$ of Beta distributions with lower mean values are greater and increase faster than that of Beta distributions with larger mean values. To be more specific, the mean of Beta distribution with shape parameters $\alpha$ and $\beta$ is $\alpha /(\alpha+\beta)=1 /(1+\beta / \alpha), \beta / \alpha$ is greater, the mean is lower and then $m(t)$ is greater. In Figure 5, the means of $\operatorname{Beta}(2,3), \operatorname{Beta}(2,2), \operatorname{Beta}(3,3)$ and $\operatorname{Beta}(3,2)$ are $0.4,0.5,0.5$ and 0.6 , respectively. It is easy to observe that their curves of $m(t)$ are in a descending way which verify our inference. Thus, to some extent, the ratio of $\beta$ and $\alpha$ that is $\beta / \alpha$ can be used to represent the burstiness of Beta distribution or that of MTC.

3) When Beta distributions have equal means, like Beta $(2,2)$ and Beta $(3,3)$, larger value of $\alpha$ and $\beta$ makes $m(t)$ smaller. This iscaused by the reduction of the variance.

All these results unanimously show us that in the context of MTC, the number of access requests from MTC devices by $t$ will be far greater than that of traditional $\mathrm{H} 2 \mathrm{H}$ communications which is usually modeled by Poisson arival. In practice, this implies increased access collisions in random access procedure and more congestion at the core network, which may seriously degrade the network perrormance. These conclusions lead to the well-known congestion problem caused by MTC and coincide with the reports in $[3,5]$.

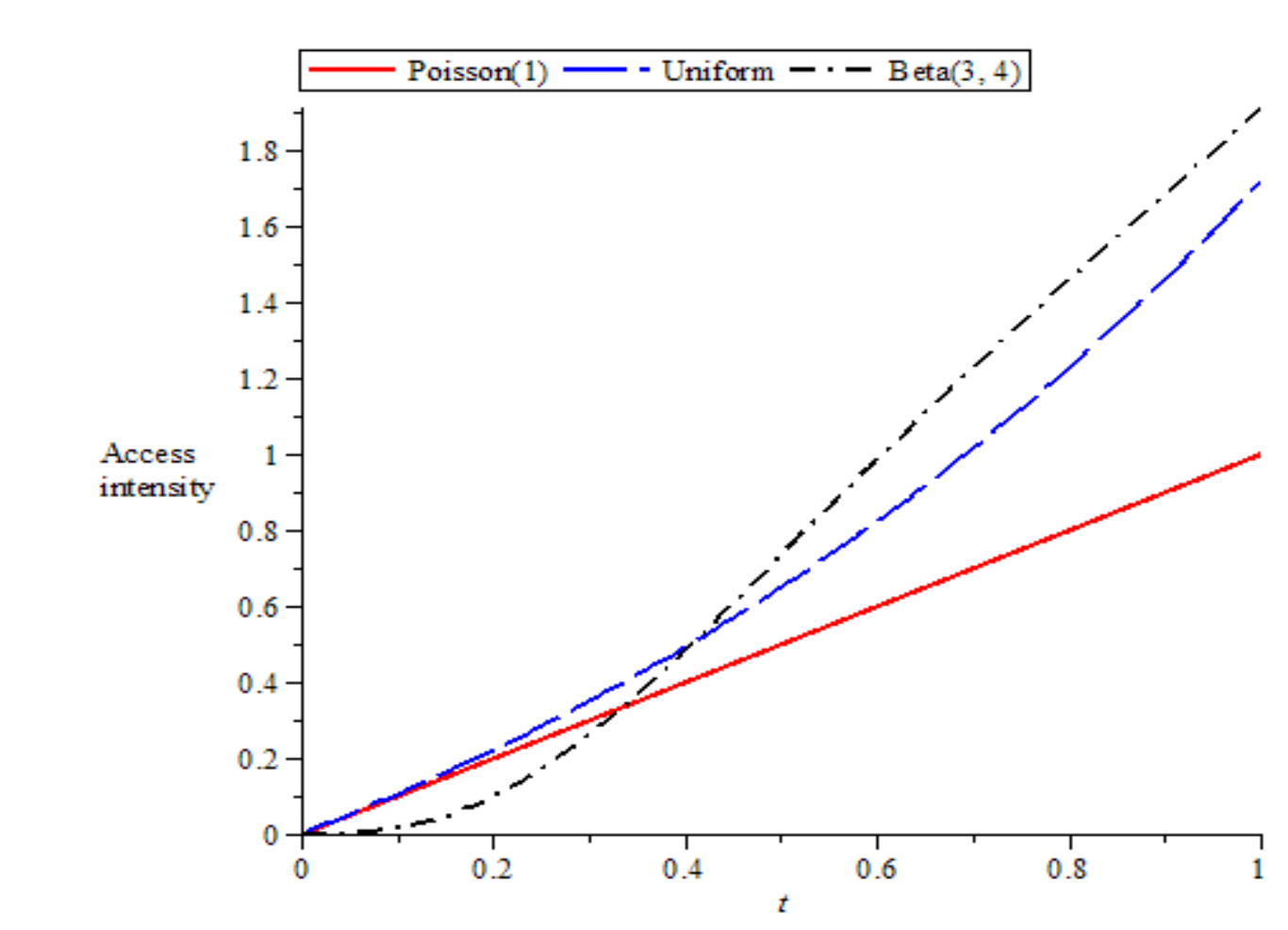

Figure 4. Access Intensity of MTC with Beta Distrbutions of 3GPP Proposals 


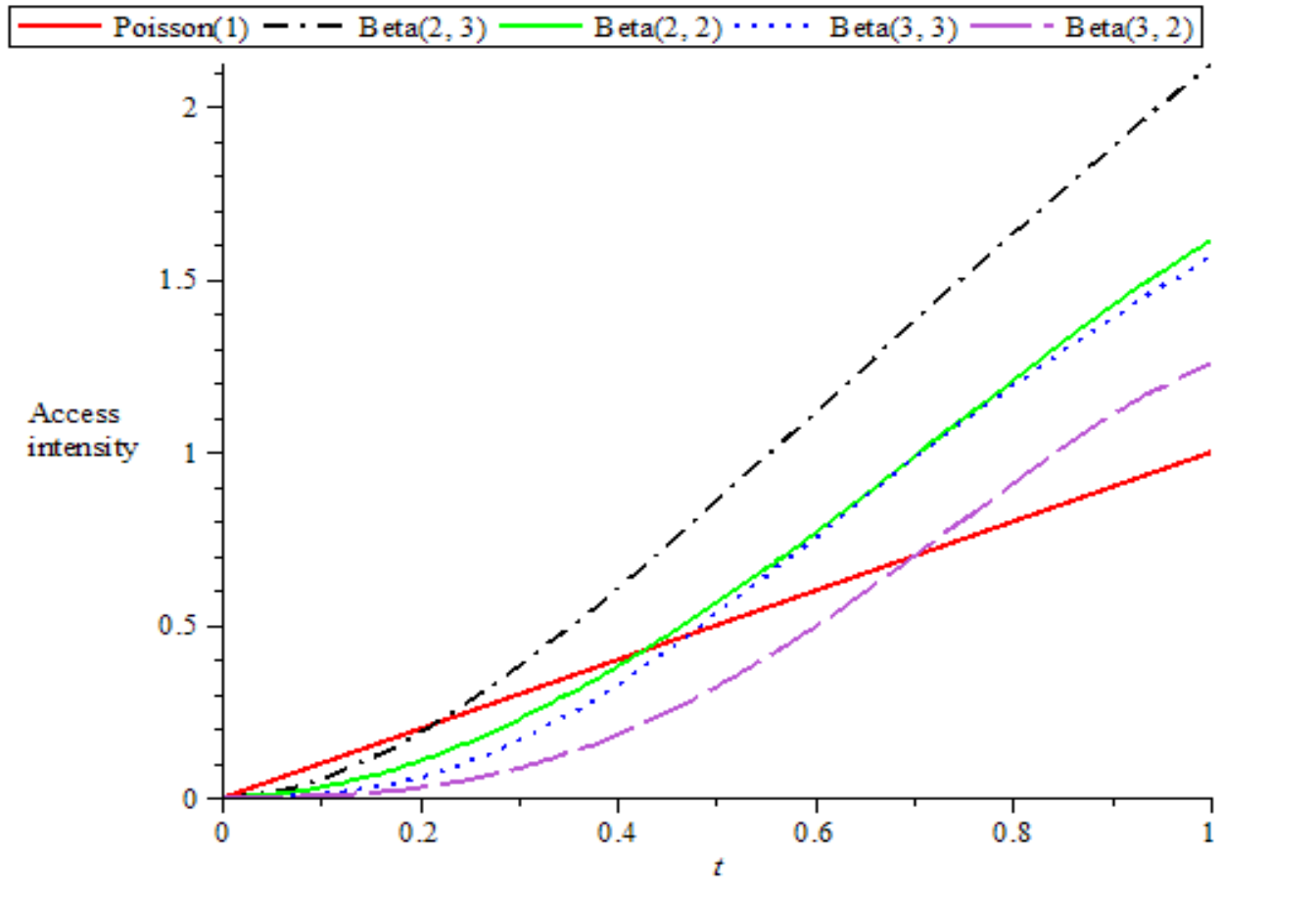

Figure 5. Access Intensity of MiTC with Low Order Beta Distributions

\section{Conclusions}

By the usage of renewal theory and Volterra integral equation of the second kind with difference kernel, we present the methodology to calculate the access intensity of MTC arrival process. Numerical results show that MTC applications will undoubtedly increase the access load of network. And the ratio of $\beta$ and $\alpha$ of $\operatorname{Beta}(\alpha, \beta)$ distribution to some extent can be used to serve as the burstiness metric of Beta distribution or that of MTC applications.

\section{Acknowledgements}

This work was supported by the National Natural Science Foundation of China (NSFC) under Grants 61171089. The authors would like to thank the anonymous referees whose insightful comments helped us to improve the presentation of the paper.

\section{References}

[1] 3GPP. Future Radio in 3GPP. 3GPP RAN Chairman workshop, (2012) June 11-12; Ljubljana, Stovenia.

[2] 3GPP TR 36.888. Study on Provision of Low Cost MTC UEs Based on LTE. (2012) June.

[3] Taleb, Tarik, and Andreas Kunz. Machine Type Communications in 3GPP Networks: Potential, Challenges, and Solutions. IEEE Communications Magazine, (2012) March Vol.50, No.3, pp. 178184.

[4] Xin Jian, Xiaoping Zeng, Li Zhang, Mengru Li, Yuan He. Service Modeling and Traffic Analysis for Machine Type Communications. Journal of Beijing University of Posts and Telecommunications, (2013) June, Vol. 36, No. 3, pp. 74-78.

[5] 3GPP TS 22.368. Service Requirements for Machine-Type Communications. (2012) February.

[6] Oliver C. Ibe. Markov Process for Stochastic Modeling. Elsevier, London (2008), pp. 105-148 
[7] Nikaein N, Laner M, Zhou K, et al. Simple Traffic Modeling Framework for Machine Type Communication. Proceedings of the Tenth International Symposium on Wireless Communication Systems, (2013) August 27-30, pp. 1-5.

[8] Laner M, Svoboda P, Nikaein N, et al. Traffic Models for Machine Type Communications [C]. Proceedings of the Tenth International Symposium on Wireless Communication Systems, (2013) August 27-30, pp. 6-10.

[9] 3GPP R2-100204.Traffic model for M2M service. Huawei, (2010) July.

[10] Wu H, Zhu C, La R J. FASA: Accelerated S-ALOHA using access history for event driven M2M communications. IEEE/ACM Transactions on Networking, (2013) December, Vol. 21, No. 6, pp.114.

[11] 3GPP TR 37.868. Study on RAN Improvements for Machine Type Communication. (2011) September.

[12] Georgii, Hans-Otto. Stochastics: Introduction to Probability and Statistics. Walter De Gruyter, Berlin (2008), pp. 42-44.

[13] Elderton, William Palin and Norman Lloyd Johnson. Systems of frequency curves. Cambridge University Press, London (1969), pp. 42-56.

[14] Sheldon M.Ross. Introduction to Probabilty Models. Elsevier, London (2010), pp. 497 568.

[15] Polyanin, Andrei D., and Alexander V. Manzhirov. Handbook of integral equations. Chapman and Hall/CRC, London (2008), pp. 544-549.

[16] Xin Jian, Xiaoping Zeng. Beta/M/1 Model for Machine Type Conmunication. IEEE Communications Letters, (2013) March, Vol. 17, No. 3, pp. 584-587

[17] Liu Shida, Liu Shishi. Special Funtion. China Meteorological Press, Beijing (1988), pp. 537-551.

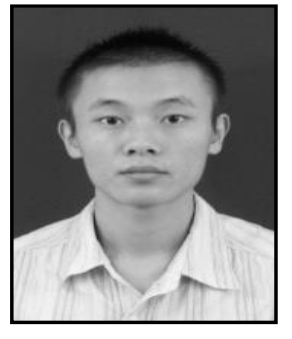

\section{Authors}

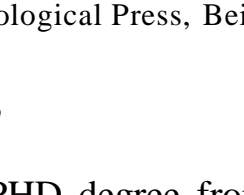

Xin Jian, received his BEPHD degree from Chongqing University, Chongqing, China inc2014. He is currently a lecture at the College of Communication Eńgineering, Chongqing University, China. His current interests include statistical learning theory, computational mathematics, network information theory and the next generation mobile communication.

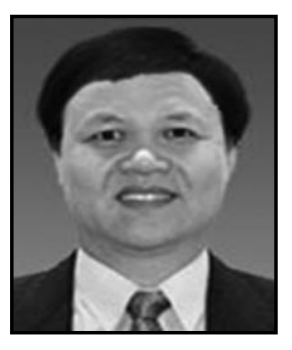

Xiaoping Zeng, received the B.E., M.S., and Ph.D. degrees in Electrical Engineering from Chongqing University, Chongqing, China in 1982, 1987, and 1996, respectively. He is now a professor and $\mathrm{Ph}$. D. supervisor at the College of Communication Engineering, Chongqing University, China. His research interests include aeronautical information network, communication signal processing and biomedical signal processing.

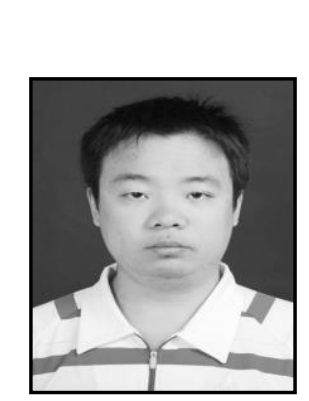

Jie Huang, received his B.E. degree from Chongqing University of Posts and Telecommunications, Chongqing, China in 2011. He is currently a Ph.D. student of five-years-educational system at the College of Communication Engineering, Chongqing University, China. His interests include internet of things, knapsack problem and cognitive radio. 


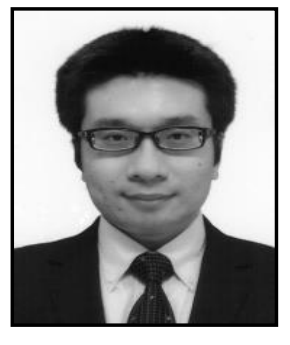

Yunjian Jia, received his B.E. degree from Nankai University, China, and his M.S. and Ph.D. degrees from Osaka University, Japan, in 1999, 2003 and 2006, respectively. From 2006 to 2012, he engaged in research and development on wireless networks at Central Research Laboratory, Hitachi, Ltd., and contributed to LTE/LTE-Advanced standardization in 3GPP. He is now a professor at the College of Communication Engineering, Chongqing University, China. His major research interests include multiple antenna technologies, radio access networks, resource management and coordinated operation in wireless networks. Dr. Jia is a member of IEEE and IEICE.

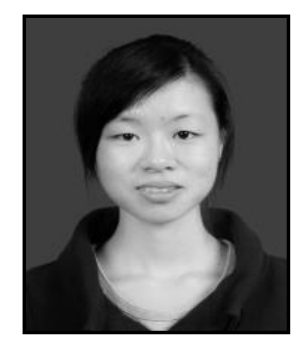

Yu Zhou, received his B.E. degree from Chongqing University, Chongqing, China in 2013. He is a master student at the College of Communication Engineering, Chongqing University, China. His interests include internet of thing and wireless communication systems such as LTE and LTE-Advanced.

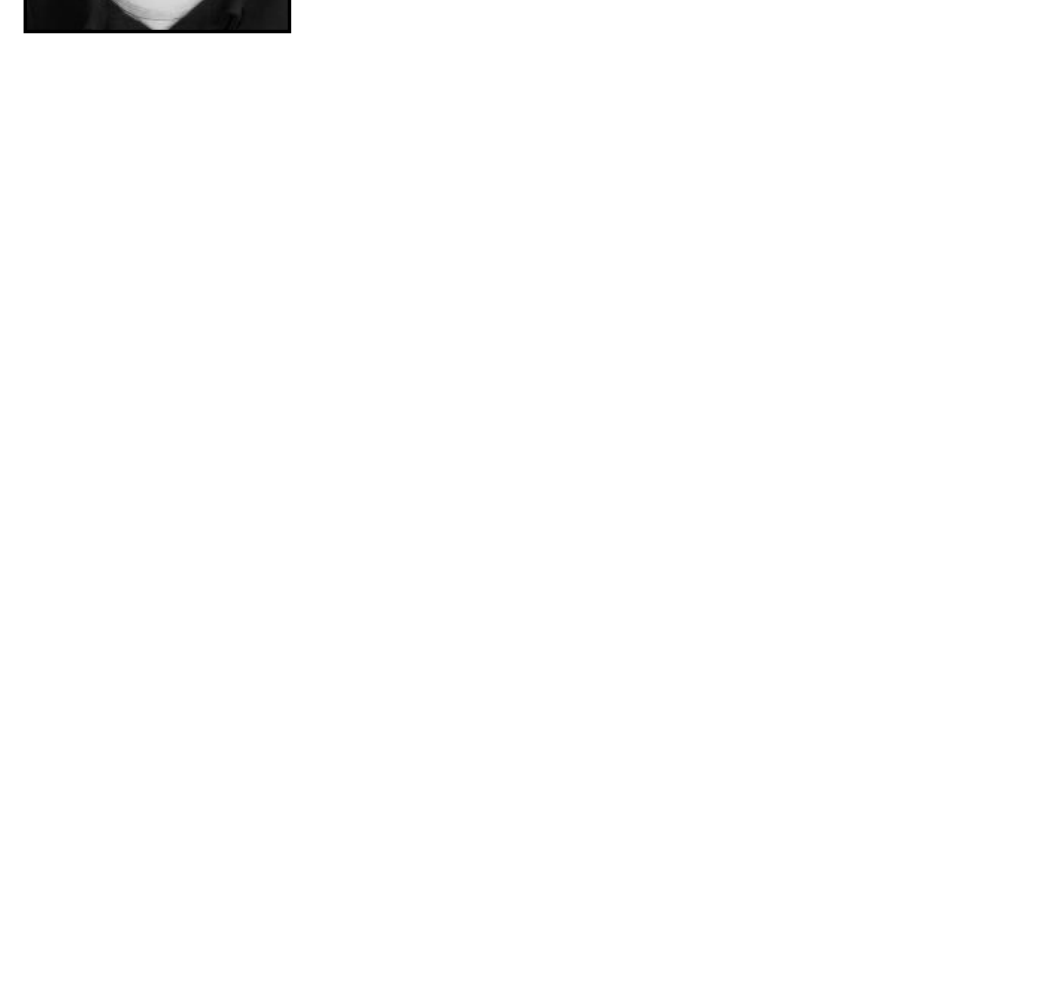


International Journal of Smart Home

Vol.8, No.4 (2014)

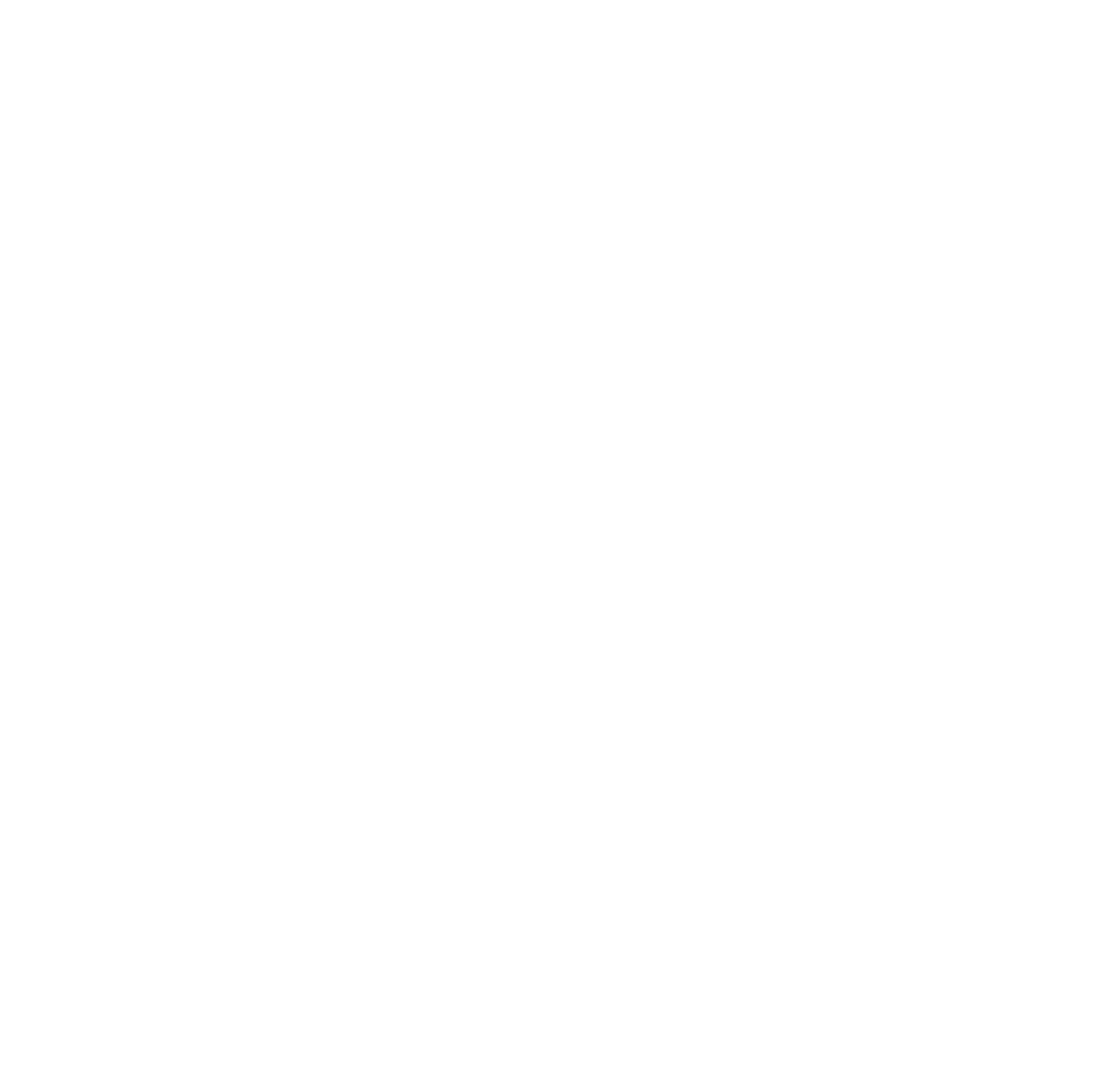

